\title{
Structural topology optimization based on improved genetic algorithm
}

\author{
Qu Dongyue ${ }^{1, a}$, Huang Yangyang ${ }^{2, b}$ and Song Jinyu ${ }^{3, c}$ \\ ${ }^{1}$ School of Harbin, engineering University, Harbin 150000, China; \\ 2 School of Harbin, engineering University, Harbin 150000, China; \\ ${ }^{3}$ School of Northeast, agricultural University, Harbin 150000, China. \\ aqudongyue@hrbeu.edu.cn, 'buangyangyang2009@163.com, 'brucesong778899@163.com
}

Keywords: Genetic algorithm, Bi-directional evolutionary structural optimization algorithm, Topology optimization.

\begin{abstract}
This article aim at solving the problem of local optimal solution easily appear in structural optimization by the algorithm of bi-directional evolutionary structural optimization (BESO), and importing improved genetic algorithm (GA), put forward a new algorithm named bi-directional evolutionary structural optimization based on improved genetic algorithm (IGA-BESO). In study, it adopts the dual coding method to build the model of the structure, through a suitable for topology optimization of crossover and mutation with penalty factor method to update the individual genes of the population. This paper, optimize a typical structure for compliance optimization with multiple load cases with method of IGA-BESO, and the optimization results show that the proposed method not only improves the computational efficiency, but also improve the stiffness of the structure.
\end{abstract}

\section{Introduction}

The most typical topology optimization method is bi-directional evolutionary structural optimization (BESO), Huang \& Xie (2007) put forward the improved BESO [1], BESO method is improved to optimize the structure, and the final optimization structure is clean, no checkerboard and gray phenomenon, easy to convergence, easy to production and process. BESO method through the element removal ratio (ER) control iteration target volume in every step, gradually remove the inefficient or invalid element. This method is heuristic control iteration, and it may change the force transfer path. So it may lead to a local solution, and unable to obtain the global optimal solution. Genetic algorithm is a kind of intelligent algorithm on the basis of the principle of "survival of the fittest", and it has a high ability to search the global optimal solution [2]. Combining BESO with improved GA, this paper puts forwards the IGA-BESO optimization algorithm. It has solved the problem of local optimal solution which may occur in BESO algorithm in the process of structural optimization [3], at the same time reducing the GA genes number of the population in the iterative process, improving the efficiency of calculation.

\section{Sensitivity Analysis}

Reference the article [4], this paper build the topology optimization model for the stiffness optimization under the multiple load cases, as shown in the formula (1).

$$
\begin{array}{cc}
\text { Minimize } & f(x)=\sum_{k=1}^{L} \omega_{k} C_{k} \\
\text { Subject to: } & \mathrm{V}^{*}-\sum_{i=1}^{n} \mathrm{~V}_{i} x_{i}=0 \\
& x_{i}=x_{\min } \text { or } 1
\end{array}
$$

In the formula (1), $\omega 1+\omega 2 \ldots+\omega \mathrm{L}=1, \omega \mathrm{k}$ is the weight of kth load case, Ck is the compliance of kth load case, $\mathrm{V}^{*}$ is the target volume, $\mathrm{Vi}$ is the volume of ith element, $\mathrm{n}$ is the number of solid elements, $\mathrm{xi}$ is the design variable. 
The sensitivity of structural optimization can be expressed as the following formula (2).

$\alpha_{i}=\frac{1}{2} x_{i}^{p-1} \sum_{k=1}^{M} \omega_{k}\left(u_{i}^{T} K_{i}^{0} u_{i}\right)_{k}$

In the formula (2), $\alpha i$ is the sensitivity of ith element, $p$ is penalty factor, Ki0is the stiffness matrix of ith solid element, ui is the displacement matrix of ith element.

\section{Improved genetic algorithm}

The process of the improved genetic algorithm for structural optimization can be shown in the figure 1 . Due to the natural randomness of genetic algorithm, it is possible to appear element disconnection phenomenon, leading to the structure cannot bear any load. In order to overcome the disconnection problem, this paper introduce the punishment mechanism [3], let the factor Pc and Pm increasing gradually to 1 so that the high sensitivity of individual genes contain more ' 1 ', and increase the ability of crossover and mutation within the same class which divided according to the sensitivity.

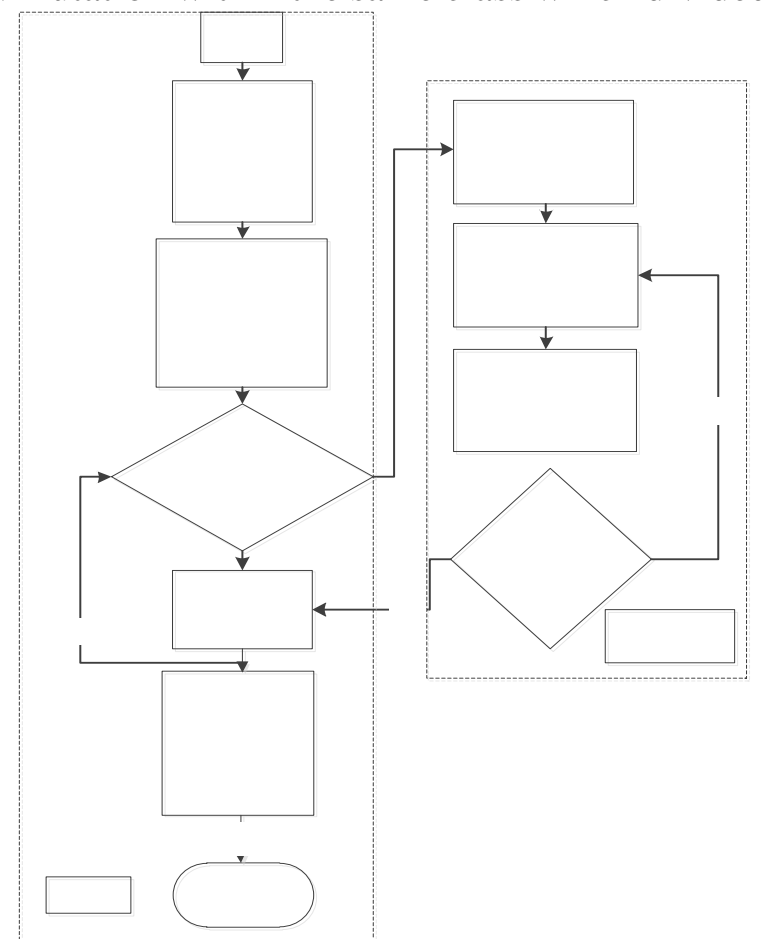

Figure. 1 IGA-BESO Structural optimization flow chart

This punishment mechanism can eliminate the phenomenon of element disconnection. The punishment mechanism of Pc and Pm as shown in the following formula (3).

$$
\begin{aligned}
& P_{c}=P_{c \text { min }}+\left(P_{c \text { max }}-P_{c \text { min }}\right) \operatorname{Pr} g^{\text {Pen }} \\
& P_{m}=P_{\text {m min }}+\left(P_{\text {mmax }}-P_{\text {m min }}\right) \operatorname{Pr} g^{\text {Pen }}
\end{aligned}
$$

In this paper, structural optimization parameters settings as shown in table 1.

Table 1 Parameter settings in the optimization process of IGA-BESO

\begin{tabular}{cc}
\hline Parameter name & Scheme 1 \\
\hline Minimum crossover probability & 0.2 \\
Minimum mutation probability & 0.1 \\
Maximum Crossover probability & 1 \\
Maximum Mutation probability & 1 \\
The length of the binary character string & 20 \\
\hline
\end{tabular}




\section{Calculation and result analysis}

(1) 2D structure of the cantilever beam

This paper analyze the typical structure of topology optimization, and compare the method BESO with IGA-BESO. The initial design area of the structure is shown in table 2 . The size of the rectangle is $70 \mathrm{~mm} \times 60 \mathrm{~mm}$, the thickness of the cantilever beam is $1 \mathrm{~mm}$. According to the finite element analysis, the design area will be divided into $70 \times 60$, the size of the element is $1 \mathrm{~mm} \times 1 \mathrm{~mm}$. The structure left end is fixed, the top and bottom of right end of the structure bear $\mathrm{F} 1=1 \mathrm{~N}$ and $\mathrm{F} 2=1 \mathrm{~N}$ concentrated loads. The Young's Modulus is $1 \mathrm{MPa}$ and Poisson's ratio is 0.3 . The object volume is $45 \%$ of the design domain with a filter radius of $5 \mathrm{~mm}$. Element removal ratio (ER) .

Table 2 Topology optimization with BESO method

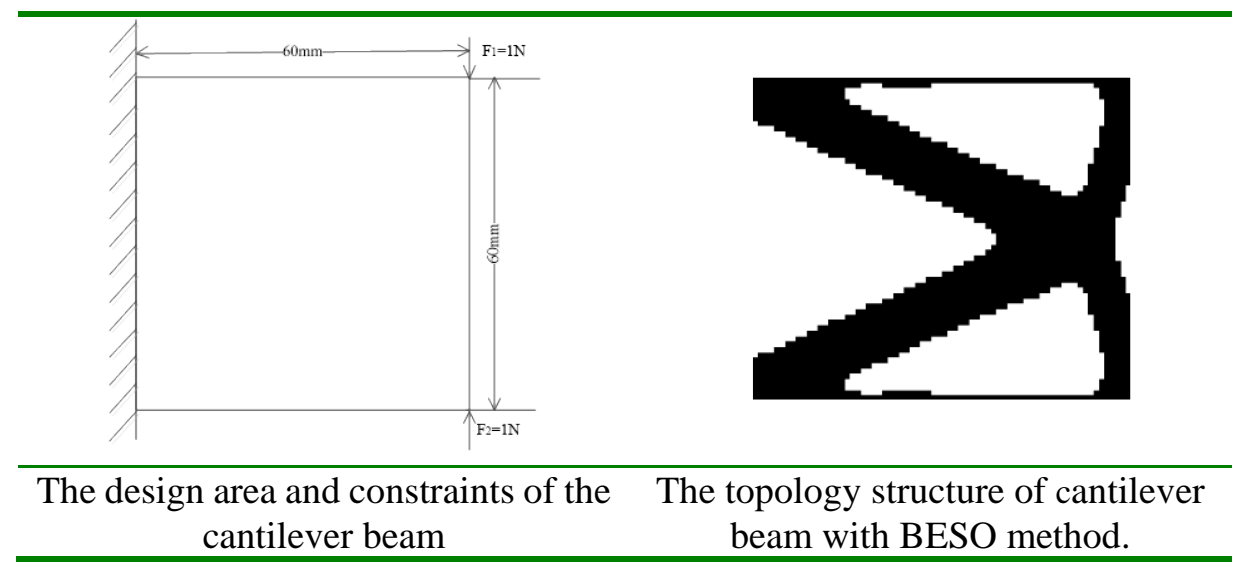

A typical method of structure optimization is BESO, cantilever beam structure for compliance optimization with multiple load cases is optimized by BESO, the value of total iteration steps and the even compliance is shown in table 4. The final optimization topology structure is shown in table 3.

Table 3 Optimization topology structure of cantilever beam with IGA-BESO method

run

Total iteration steps and even compliance with BESO method can be seen form table 4.

Table 4 Total iteration steps and even compliance with BESO method

\begin{tabular}{cc}
\hline total iteration steps & 100 \\
\hline Even compliance $(\mathrm{N} \cdot \mathrm{mm})$ & 100.9141 \\
\hline
\end{tabular}

Total iteration steps and even compliance with IGA-BESO method can be seen form table 5. 
Table 5 Total iteration steps and even compliance with IGA-BESO method

\begin{tabular}{cccccccc}
\hline Calculation times & 1 & 2 & 3 & 4 & 5 & 6 & $\begin{array}{c}\text { Even } \\
\text { value }\end{array}$ \\
\hline $\begin{array}{c}\text { total iteration steps } \\
\begin{array}{c}\text { Even compliance } \\
(\mathrm{N} \cdot \mathrm{mm})\end{array}\end{array}$ & 98.4594 & 98.6578 & 98.4594 & 98.3737 & 98.6578 & 98.4594 & 90.1 \\
\hline
\end{tabular}

Analyze the experimental results can be found that the calculation value of total iteration steps and even compliance at six times is stable, more importantly, the final topology structure of IGA-BESO method is clean, no checkerboard and gray phenomenon like BESO method, it is confirmed that the IGA-BESO method is reliability and stability.

The even total iteration steps of IGA-BESO is only 60.1, but 100 for BESO, the former reduces about $40 \%$ compare with the latter, so larger model for optimization with IGA-BESO method can save time. It can be seen form the table 5 and table 4, even compliance of structure is $98.5113 \mathrm{~N} \cdot \mathrm{mm}$ with the method of IGA-BESO, but $100.9141 \mathrm{~N} \cdot \mathrm{mm}$ with BESO. In conclusion, the proposed IGA-BESO method not only improves the computational efficiency, but also improve the stiffness of the structure.

(2) 2D structure of the beam

This paper optimize the structure of beam for compliance optimization with multiple load cases with the method of IGA-BESO. The initial design area of the structure is $100 \mathrm{~mm} \times 50 \mathrm{~mm}$ as shown in figure 2, constraints $\mathrm{X}$ and $\mathrm{Y}$ direction degrees of freedom on the center of two ends, the structure bear concentrated loads $F=1 \mathrm{~N}$ at the up face and down face shown in the picture. The Young's Modulus is $1 \mathrm{MPa}$ and Poisson's ratio is 0.3 .

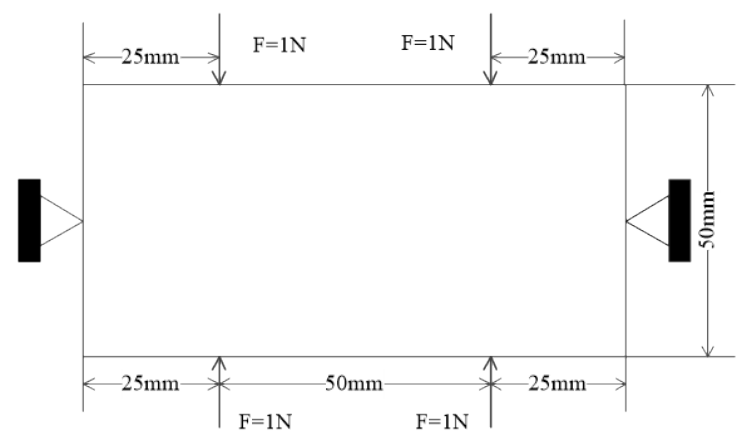

Fig. 2 Design area and constraints of the beam.

Table 6 shows topology structure of two dimensional beam in the six iterations using the same parameters. The topology optimization structure of the beam in six iteration are almost the same,so the IGA-BESO method has stability and reliability.

Table 6 Optimization topology structure with IGA-BESO method of beam

(n)


Table 7 Total iteration steps and even compliance with IGA-BESO method

\begin{tabular}{cccccccc}
\hline Calculation times & 1 & 2 & 3 & 4 & 5 & 6 & Even value \\
\hline total iteration steps & 32 & 33 & 34 & 32 & 33 & 34 & 33.1 \\
$\begin{array}{c}\text { Even compliance } \\
\text { (N.mm) }\end{array}$ & 31.5092 & 31.3012 & 31.1885 & 31.5092 & 31.3612 & 31.1885 & 31.3430 \\
\hline
\end{tabular}

Table 7 shows the even compliances and total number of iterations of two dimensional beam under the compliance optimization with multiple load cases. Even compliance changes relatively stable with average total iteration number 33, and optimization speed is faster, mainly due to at the begin of structural optimization can make the low sensitivity of individual genes contains more numbers of ' 0 ' character in genes through individual genetic crossover and mutation according to the sort of element sensitivity and quickly remove inefficient or invalid element, thus can save time and space for subsequent optimization.

(2) 3D structure of the cantilever beam

IGA-BESO method can be applied to the three-dimensional structure of the optimization problem, this paper optimize the typical structure of 3D cantilever beam for the compliance optimization with multiple load cases. Design area and load constraints is shown in table 8. The Young's Modulus is 200GPa and Poisson's ratio is 0.3 , and the object volume is $45 \%$.

Table 8 Topology optimization with BESO method

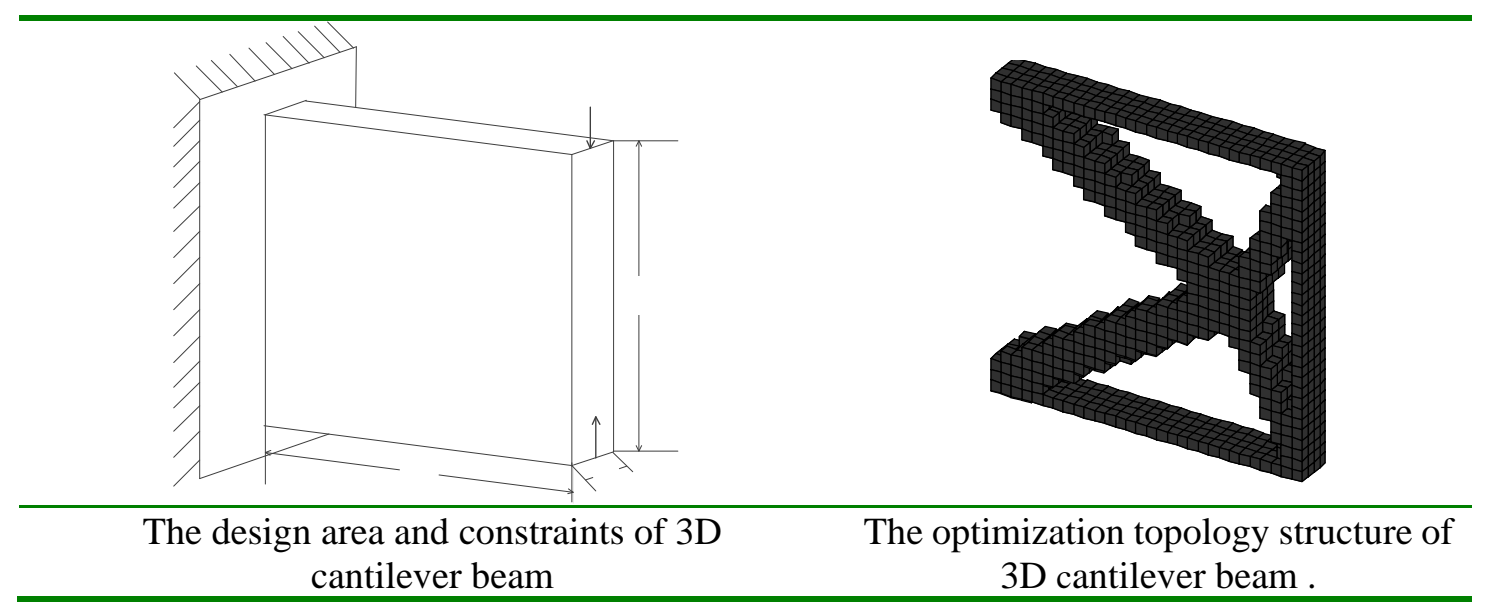

The average total iteration number is 100 , even compliances of the final structure is $68.3896 \mathrm{~N} \cdot \mathrm{m}$. Introducing the improved GA to BESO, not only solve the problem of local optimal solution in the process of optimization with BESO method, but also solve the complicated problem in GA caused by genes increase with iteration.

\section{Summary}

In this paper, optimize structures for compliance optimization with multiple load cases with the method of IGA-BESO, and it can get the following conclusion:

(1) IGA-BESO method in the optimization design can improve the compliance of structure. (2) IGA-BESO method can delete the inefficient elements quickly at the beginning of optimization. So it can save time and improve the efficiency of optimization with the method of IGA-BESO. (3) IGA-BESO method can overcome the local optimal solution occasional appears with BESO method. 


\section{References}

[1] Huang, X \& Xie, Y.M. 2007. Convergent and mesh-independent solutions for the bi-directional evolutionary structural optimization method. Finite Elem Anal Des 43:1039-1049.

[2] Wang, S.Y\& Tai, K. 2005. "Structural Topology Design Optimization Using Genetic Algorithm with a Bit-array Representation," Computer Methods in Applied Mechanics and Engineering, Vol. 194,No. 36-38, pp. 3749-3770.

[3] Zuo, Z.H, Xie, Y.M. \& Huang, X. 2009. Combining Genetic Algorithms with BESO for Topology Optimization Algorithm. Applied Soft Computing, 9(4): 1343-1353.

[4] Huang X, Xie Y M. 2009. Evolutionary Topology Optimization of Continuum Structures: Methods and Applications. United Kingdom: John Wiley \& Sons Limited. 